\title{
E-MRS Spring Meeting in June to Focus on Electronic Materials
}

According to the general goals of the European Materials Research Society (EMRS), the traditional Spring Meeting will be held in Strasbourg June 16-19, 1998 with special emphasis on "Electronic Materials." Under the auspices of the Conference Chairs P. Glasow (Germany) and Y.I. Nissim (France), 14 Symposia are planned ranging from fundamental aspects of defects in silicon to materials aspects in microsystem technologies. Forty-five scientists worldwide will serve as symposium organizers, indicating an international character of the meeting.

The symposium "Defects in Silicon: Hydrogen," organized by $\mathrm{J}$. Weber and A. Mesli, is devoted to the fundamental aspects of defects in crystalline silicon and their influence on silicon technology. The forum will stimulate interactions among researchers and technologists. It addresses particularly the recent developments in understanding the properties of hydrogen and its interaction with other defects.

F. Priolo, J. Linnros, and L. Canham organized the Symposium "Light Emission from Silicon: Progress Toward Si-Based Optoelectronics." Light emission from silicon is a rapidly expanding field attracting materials researchers worldwide. Silicon is the dominating material in microelectronics, partly due to its unrivaled properties, but also as a result of the enormous research and development invested in silicon technology. Since waveguides, modulators, and detectors have already been demonstrated in $\mathrm{Si}$, one of the major limiting step toward silicon-based optoelectronics is the achievement of efficient, stable, very large scale integration (VLSI) compatible light sources. Recently, encouraging results have been obtained in several fields comprising porous silicon, silicon-rich silicon dioxide, nanoparticles, multilayers, rare-earth-doped silicon, and iron disilicide. This forum will elicit discussion among researchers working on various approaches to obtain light from silicon or from silicon-compatible materials, and will therefore provide an opportunity to compare different technologies, their different mechanisms for light emission, and prospective application areas. Basic materials aspects in terms of preparation, materials properties, and theory as well as device fabrication and performances will be discussed.

The Symposium "Growth, Characterization and Applications of Bulk II-VI's," organized by R. Triboulet, G. MullerVogt, and P. Capper, is to bring together crystal growers, chemists, physicists, and materials scientists to discuss problems dealing with the growth, physics, and applications of II-VI's, the understanding of which is the key to any industrial application of II-VI materials, especially CdTe, ZnSe, and $\mathrm{ZnO}$.

E. Kasper and K.L. Wang organized the Symposium "Thin Films Epitaxial Growth and Nanostructures." Nanometer structures attract high scientific and technological attention by tailoring material properties artificially. Epitaxial growth is a promising way to produce low dimensional structures with nanometer dimensions for a variety of materials. This symposium will focus on microelectronics applications, semiconducting films, and on the basic understanding of interface formation and formation of nanostructures.

The Symposium "Thin Film Materials for Large Area Electronics," organized by B. Equer, B. Drevillon, I. French, and T. Kalfass, will address the area of active matrix in light-emitting diodes (LCDs), which is currently the largest application of large area electronics. Alternative display technologies (such as field emission display [FED]) are also important. Other uses such as large area sensors, like x-ray sensors, will be considered. The symposium will also emphasize poly- and microcrystalline silicon for thin-film transistors (TFTs). Other materials and processes related to TFT displays, FEDs, and sensors will also be included, as well as the topics of insulating and protective layers.

H. Richter, P. Wagner, and G. Ritter organized the symposium "Techniques and Challenges for $300 \mathrm{~mm}$ Silicon: Processing, Characterization, Modeling and Equipment." Integrated circuit production employs silicon substrates 200 $\mathrm{mm}$ in diameter. Currently, the semiconductor industry is preparing a worldwide transition to $300 \mathrm{~mm}$ silicon wafers. Demand for the larger wafers is expected to go from the pilot level in 1998 to $33 \%$ of total silicon demand by 2003 . The transition is expected to cost equipment makers and materials suppliers more then $\$ 14$ billion, creating the most expensive industrial retooling in history. Currently $300 \mathrm{~mm}$ wafers are available and first equipment is under evaluation. At present, effort is concentrated on $300-\mathrm{mm}$ complementary metal oxide semiconductor (CMOS) technology with $0.25 \mu \mathrm{m}$ feature sizes.

The challenges for $300-\mathrm{mm}$ silicon include the growth of large diameter silicon single crystals, the preparation of $300-\mathrm{mm}$ wafers, and the transition of all CMOS processes from $200 \mathrm{~mm}$ to $300 \mathrm{~mm}$. Due to higher requirements of the advanced technologies, new equipment and optimized processes are necessary. The aim of this symposium is to provide a state-of-the-art overview of the subject and to bring together materials scientists from non-industrial research centers and researchers/developers from the industrial suppliers of semiconductor materials, equipment, and chips.

The Symposium "Surface Processing: Laser, Lamp, Plasma" is organized by J. Perriere, M Stuke, I.W. Boyd, and U. Biermann. Laser, lamp, and plasma sources are particularly powerful tools for modifying and structuring surfaces and producing thin films. Applications of such surface processing methodologies are important in various fields such as microelectronics, optics, biomaterials, sensors, and actuator displays. Advances in the theoretical understanding of the underlying physical and chemical mechanisms may also lead to novel techniques in materials science. This symposium will provide an interdisciplinary discussion of recent advances in research, development, and applications of surface processing. Major subjects of the meeting include fundamental process studies, modeling of growth mechanisms and surface modification, characterization of synthesized or modified materials, material modification at a nanometric scale, development of in situ analytical techniques, and applications toward device fabrication.

The Symposium "Materials Aspects in Microsystem Technologies," organized by D. Barbier, W. Lang, D. Esteve, J.R. Morante, and G. Müller, will cover the field of microelectromechanical systems (MEMS), sensors actuators, and microstructures. The microsystem technology now experiences a fast development which highly benefits from new advances in the field of functional materials and microstructure. Progress has been made in the deposition of sensing or actuating layers, micromachining and characterization of microstructures, and the packaging and bonding of chips modules. This symposium provides a special focus on the materials processing aspects of microstructuring, which have a strong impact in microsystem manufacturing. Thus, papers giving the latest information on research and development in the field of sensitive transducing or functional materials, new processing steps, and new characterization means for layered and micromachined structures are solicited. The topics of the symposium will especially address domains where materials aspects is a key issue for the microsystem technology, such as biomedical monitoring, biochemical measurements, optical 
telecommunications, and mechanical control in harsh environments.

The Symposium "Rapid Thermal Processing," organized by A. Slaoui, J.C. Muller, and R.K. Singh, covers RTP as an emerging single-wafer technology in ultralarge-scale integration semiconductor manufacturing, electrical engineering, and materials science. Traditional RTP processes such as annealing, silicidation, deposition of dielectrics or active layers will be covered. Special emphasis is placed on equipment design of RTP systems and process monitoring and control. Another main topic will concern the potential applications of RTP in integrated technology and large area devices such as flat-panel displays and solar cells.

J.K.N. Lindner, P.L.F. Hemment, H.A. Atwater, and B. Svensson organized the Symposium "Ion Implantation into Semiconductors, Oxides and Ceramics." Ion implantation has become an indispensable tool to tailor the properties of semiconductors as well as oxidic and ceramic materials used in semiconductor device technology. Continued research covering the energy range from a few $\mathrm{keV}$ up to several $\mathrm{MeV}$ has enabled the application of ion implantation to control precisely the compositional, structural, and electrical properties of materials from near-surface regions down to depths of several microns. Shrinking semiconductor device dimensions and new device concepts using classical semiconductor materials such as silicon or $\mathrm{GaAs}$ and related insulators (e.g., $\mathrm{SiO}_{2}, \mathrm{Si}_{3} \mathrm{~N}_{4}$ ) as well as the availability of new semiconductors like $\mathrm{SiGe}, \mathrm{SiC}, \mathrm{GaN}$, and $\mathrm{AlGaN}$ on various substrates (e.g., $\mathrm{Si}, \mathrm{SiO}_{2}, \mathrm{Al}_{2} \mathrm{O}_{3}$, $\mathrm{TiO}_{2}, \mathrm{LiGaO}_{2}$ ) have triggered additional interest in the study of beam interactions with such materials. Moreover, the possibility of growing precipitate layers for optical applications and semiconducting/ insulating layer structures by ion beam synthesis has led to enormous research activities in ion implantation.
J. Fink is the organizer of the Symposium "Carbon-Based Materials for Microelectronics," which will provide an overview of the current status of the wide field of carbon-based materials. Papers will cover research and development in the fields of amorphous carbon films, undoped and doped diamond films, physics and chemistry of fullerenes, carbon nanotubes, and carbon-based nanotechnology, with special emphasis on novel materials and their application in microelectronics. The Symposium will also inform European industries about the possible application of advanced carbonbased materials in electronic devices.

The Symposium "Nitrides and Related Wide Bandgap Materials," organized by J.Y. Duboz, F.A. Ponce, A. Hangleiter, and K. Kishino, follows the rapid expansion of the subject of nitrides. This wide bandgap semiconductor family including GaN, AIN, InN, BN and related compounds has been attracting a great deal of attention, and this Symposium will address the most recent experimental and theoretical progress. Papers will provide the latest information on research and development in the fields of crystal growth, structural, optical and electrical properties of the materials, and also on the processing, characterization, and performance of nitride devices. Particular topics to be addressed include growth of bulk materials and thin films by metalorganic chemical vapor deposition, molecular-beam epitaxy, and other techniques; substrates; strain and defects; alloys with special attention to the problem of InGaN growth; doping issues; optical properties including excitons and biexcitons; electrical properties; heterostructures and quantum wells; quantum wires and dots; and optical pumping and emission mechanisms. Special emphasis will also be laid on devices: processing, etching, ohmic and Schottky contacts, photodetectors and modulators, cold cathodes, bipolar and field effect transistors, lightemitting diodes, and lasers.
J. Zyss, F. Garnier, and F. Agranovich are the organizers of the Symposium "Molecular Photonics for Optical Telecommunications: Materials, Physics and Device Technology." The development of broadband telecommunication networks depends on progress in materials and devices capable of generating, routing, and processing ever increasing flows of optical data. In this respect, organic materials are increasingly emerging as appealing and realistic alternatives to purely inorganic materials-based devices toward optical signal processing and communication functions. The symposium will focus on devices for photonics and related communication issues. State-of-the-art fundamental and applied issues in the areas of molecular engineering, physics, fabrication techniques, characterization, and application of organics targeted toward photonics, optoelectronics, and related telecommunications-oriented applications will be addressed. Special emphasis will be put on reliability properties of particular relevance to bridge the gap between early demonstrative breakthroughs and actual industrial implementation.

The Symposium "Materials and Processes for Submicron Technologies" is organized by J.M. Martinez-Duart, R.A. Levy, and R. Madar. Advances in microelectronic devices and information technologies are directly related to the reduction in the size of electronic devices and interconnections down to the submicron size. This Symposium will emphasize materials and processes for submicron silicon devices, although some topics in other technologies (III-V, II-VI compounds) or even quantum structures and single electron devices are covered.

The preregistration deadline is May 18, 1998. For more information on the meeting, contact E-MRS Spring Meeting, BP 20, F 67037 Strasbourg, Cedex 2, France; 33-3-88-10-65-43; fax 33-3-88-10-62-93; e-mail emrs@phase.c-strasbourg.fr.

H.U. HABERMEIER Max-Plank Institute, StUTtgart

\title{
MRS BULLETIN SEEKS IUMRS NEWS ON
}

- Upcoming Meetings

- Conference Reports

- Important Technical Developments
- Research News

- Policy Issues

- Educational Initiatives

\author{
MRS Bulletin, Materials Research Society \\ 506 Keystone Drive, Warrendale, PA 15086-7573 USA \\ E-mail: bulletin@mrs.org, Fax: 724-779-8313
}




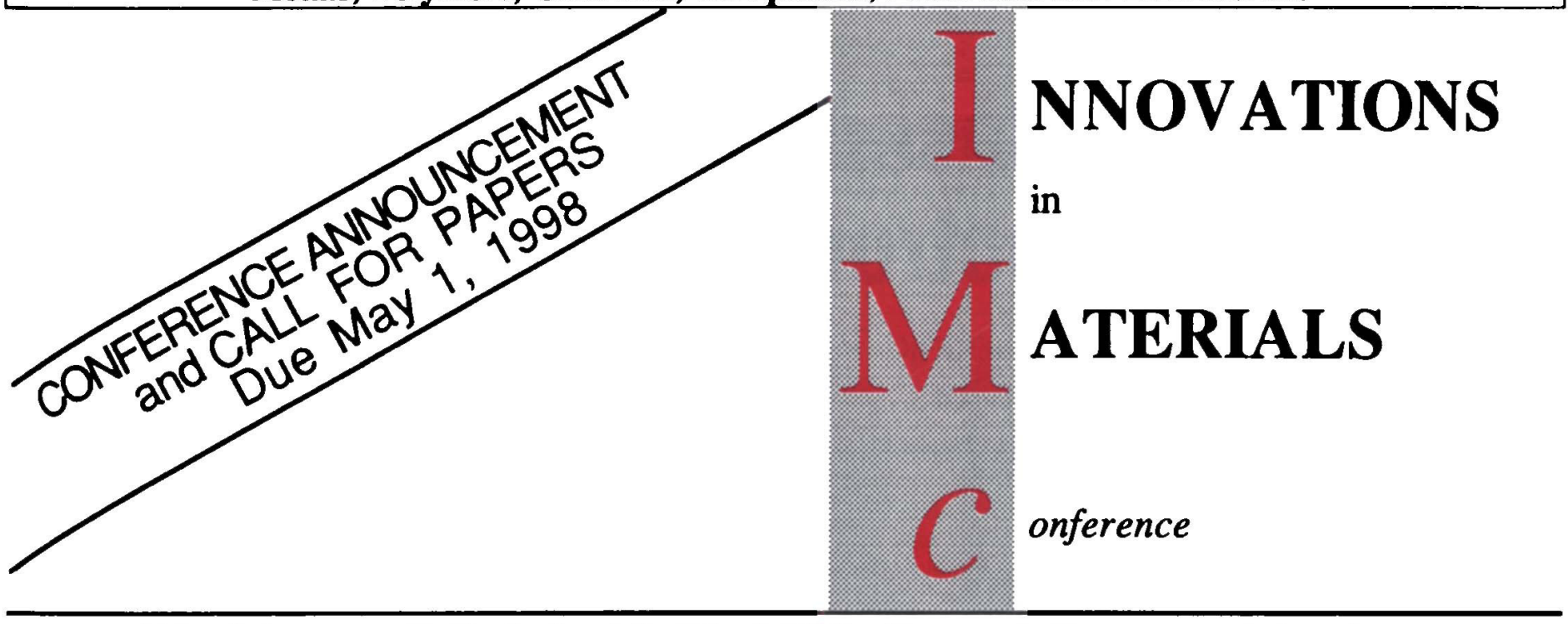

Washington DC, July 19-22, 1998, Crystal Gateway Marriott

Sponsored by International Union of Materials Research Societies (IUMRS)

IMc is a UNIQUE CONFERENCE in the field of MSE. It focuses only on step-function advances leading to: New materials with real world impact.

Papers describing unexpected results on innovations in any aspect of materials research, engineering, and development are invited, including:

- synthesis of new phases or composites

- new processes for making materials faster, cheaper, or greener

- new characterization tools and their application

- new theories to explain new observations

- new devices based on new materials

Papers submitted will be published in the new archival super-peer reviewed Journal, Materials Research Innovations by Springer-Verlag.

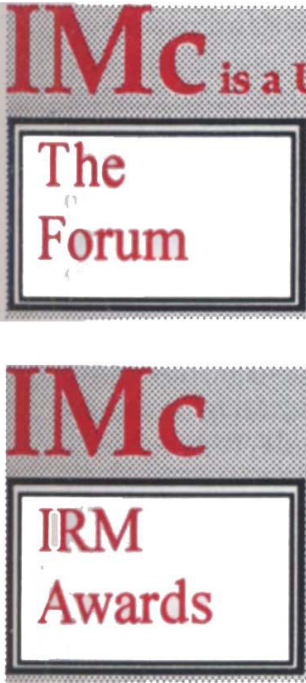

Nominated by members of various National Academies, a dozen papers will be selected for the Innovations in Real Materials Awards, which honor the most significant advances in Materials, which can affect technology and society. A special "Lifetime of Innovations" Award will be given to Dr. Kazuo Inamori, Chairman of Kyocera Industrial Ceramics Corporation. 